University of Nebraska - Lincoln

DigitalCommons@University of Nebraska - Lincoln

$12-2006$

\title{
Modulational Instability in a Layered Kerr Medium: Theory and Experiment
}

\author{
Martin Centurion \\ University of Nebraska-Lincoln, martin.centurion@unl.edu \\ Mason A. Porter \\ California Institute of Technology \\ Ye Pu \\ California Institute of Technology, yepu@sunoptics.caltech.edu \\ P. G. Kevrekidis \\ University of Massachusetts, Amherst \\ D. J. Frantzeskakis \\ University of Athens (Greece)
}

See next page for additional authors

Follow this and additional works at: https://digitalcommons.unl.edu/physicscenturion

Part of the Physics Commons

\footnotetext{
Centurion, Martin; Porter, Mason A.; Pu, Ye; Kevrekidis, P. G.; Frantzeskakis, D. J.; and Psaltis, Demetri, "Modulational Instability in a Layered Kerr Medium: Theory and Experiment" (2006). Martin Centurion Publications. 10.
}

https://digitalcommons.unl.edu/physicscenturion/10

This Article is brought to you for free and open access by the Research Papers in Physics and Astronomy at DigitalCommons@University of Nebraska - Lincoln. It has been accepted for inclusion in Martin Centurion Publications by an authorized administrator of DigitalCommons@University of Nebraska - Lincoln. 


\section{Authors}

Martin Centurion, Mason A. Porter, Ye Pu, P. G. Kevrekidis, D. J. Frantzeskakis, and Demetri Psaltis 


\title{
Modulational Instability in a Layered Kerr Medium: Theory and Experiment
}

\author{
Martin Centurion, ${ }^{1,2}$ Mason A. Porter, ${ }^{2,3}$ Ye Pu, ${ }^{1}$ P. G. Kevrekidis, ${ }^{4}$ D. J. Frantzeskakis, ${ }^{5}$ and Demetri Psaltis ${ }^{1}$ \\ ${ }^{1}$ Department of Electrical Engineering, California Institute of Technology, Pasadena, California 91125, USA \\ ${ }^{2}$ Center for the Physics of Information, California Institute of Technology, Pasadena, California 91125, USA \\ ${ }^{3}$ Department of Physics, California Institute of Technology, Pasadena, California 91125, USA \\ ${ }^{4}$ Department of Mathematics and Statistics, University of Massachusetts, Amherst, Massachusetts 01003-4515, USA \\ ${ }^{5}$ Department of Physics, University of Athens, Panepistimiopolis, Zografos, Athens 15784, Greece
}

(Received 29 July 2006; published 7 December 2006)

\begin{abstract}
We present the first experimental investigation of modulational instability in a layered Kerr medium. The particularly interesting and appealing feature of our configuration, consisting of alternating glass-air layers, is the piecewise-constant nature of the material properties, which allows a theoretical linear stability analysis leading to a Kronig-Penney equation whose forbidden bands correspond to the modulationally unstable regimes. We find very good quantitative agreement between theoretical, numerical, and experimental diagnostics of the modulational instability. Because of the periodicity in the evolution variable arising from the layered medium, there are multiple instability regions rather than just one as in a uniform medium.
\end{abstract}

PACS numbers: 05.45.Yv, 42.65.Sf, 42.65.Tg

Introduction. - The modulational instability (MI) is a destabilization mechanism for plane waves. It leads to delocalization in momentum space and, in turn, to localization in position space and the formation of solitary-wave structures. The MI arises in many physical contexts, including fluid dynamics [1], nonlinear optics [2], plasma physics [3], Bose-Einstein condensate (BEC) physics [4], and so on.

The MI was originally analyzed in uniform media, mainly in the framework of the nonlinear Schrödinger equation. There, MI occurs for a focusing nonlinearity and long-wavelength perturbations of the pertinent plane waves [1-4]. Recently, several experimentally relevant settings with (temporally and/or spatially) nonuniform media have emerged. Such research includes the experimental observation of bright matter-wave soliton trains in BECs induced by the temporal change of the interatomic interaction from repulsive to attractive through Feshbach resonances [5]. This effective change of the nonlinearity from defocusing to focusing leads to the onset of MI and the formation of the soliton trains [6]. Soliton trains can also be induced in optical settings (e.g., in birefringent media [7]). Even closer to this Letter's theme of periodic nonuniformities is the vast research on photonic crystals [8] and the experimental observations of the MI in spatially periodic optical media (waveguide arrays) [9] and BECs confined in optical lattices [10]. Finally, apart from the aforementioned results pertaining to systems that are periodic in the transverse dimensions, there exist physically relevant situations for which the periodicity is in the evolution variable. Examples were initially proposed in the context of optics through dispersion management [11] and have since also been studied for nonlinearity management both in optics $[12,13]$ and BECs [14].

In this Letter, we present the first experimental realization of MI in a setting where the nonlinearity is periodic in the evolution variable, which here is the propagation distance. There is a fundamental difference between such a periodic setting and a uniform one: In the latter, there is a cutoff wave number above which MI is not possible. In other words, there is a single window of unstable wave numbers. In a periodic medium, however, additional instability windows exist for wave numbers above the first cutoff. Our experiments were designed to demonstrate this unique feature of the layered structure. In addition to our experiments, our investigation includes a linear stability analysis $[15,16]$, which leads to a Hill equation (whose coefficients are periodic in the evolution variable) [17]. The permissible spectral bands of this equation correspond to modulationally stable wave numbers, and the forbidden bands indicate MI. The obtained experimental and analytical results are also corroborated by numerical simulations.

Our setup consists of an optical medium with periodically alternating glass and air layers. The piecewiseconstant nature of the material coefficients leads to a linear stability condition (for plane waves) along the lines of the Kronig-Penney model of solid state physics [18] (generalizations of which with spatially periodic nonlinearity have been considered in [19]). This allows us to compute the MI bands analytically and to compare the experimental findings with the theoretical predictions.

Experimental setup. - In our experiments (see Fig. 1), an amplified titanium:sapphire laser is used to generate 150-fs pulses with an energy of $2 \mathrm{~mJ}$ at a wavelength of $\lambda=$ $800 \mathrm{~nm}$. The beam profile is approximately Gaussian with a full width at half maximum of $1.5 \mathrm{~mm}$. The laser pulses are split into a pump and a reference using a beam splitter (BS1), with most of the energy in the pump pulse. After synchronization with a variable delay line, the two pulses are recombined at a second beam splitter (BS2) and sent to the periodic nonlinear medium (NLM). The reference introduces a sinusoidal modulation in the intensity (i.e., an 


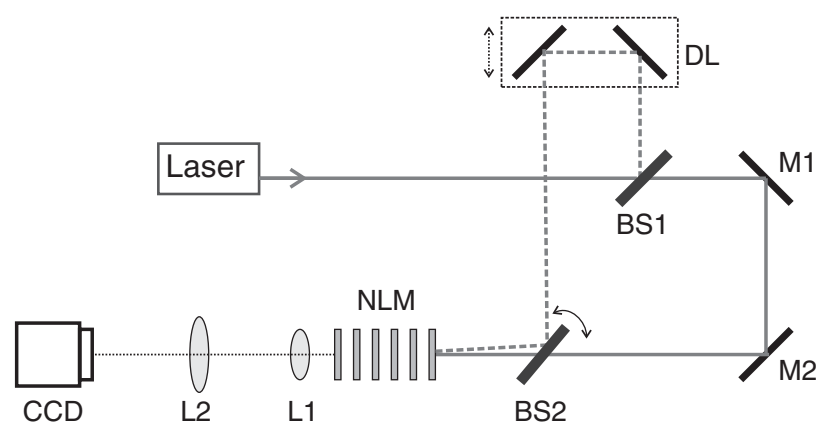

FIG. 1. Experimental setup. BS1 and BS2 are beam splitters, DL is a variable delay line, M1 and M2 are mirrors, NLM is the layered nonlinear medium, and L1 and L2 are lenses.

interference pattern), with the period determined by the relative angle between the two beams. The angle of the reference is carefully tuned by rotating BS2 so that the two beams overlap while propagating through the NLM at adjustable angles. The NLM consists of six $1 \mathrm{~mm}$ thick quartz slides separated by air gaps. The glass slides have an antireflection coating to minimize the loss (the reflection from each interface is $1 \%$ ). The loss due to backreflections from the slides is included in our numerical simulations below, and the effect of double reflections is negligible. In our experiments, we used structures with air gaps of 2.1 and $3.1 \mathrm{~mm}$. The intensity pattern after the NLM (at the output face of the last quartz slide) is imaged on a CCD camera (Pulnix TM-7EX) using two lenses (L1 and L2) in a 4-F configuration with a magnification of $M=8$. The $\mathrm{CCD}$ camera captures the central region $(0.6 \mathrm{~mm} \times$ $0.8 \mathrm{~mm}$ ) of the beam.

The intensity pattern at the output of the NLM is recorded both for a high pump intensity $\left(I_{\mathrm{P} 1}=1.3 \times\right.$ $\left.10^{11} \mathrm{~W} / \mathrm{cm}^{2}\right)$ and a low pump intensity $\left(I_{\mathrm{P} 2}=\right.$ $9 \times 10^{8} \mathrm{~W} / \mathrm{cm}^{2}$ ). In both cases, the intensity of the reference beam is $1 \%$ of that of the pump. We measure the effect of the nonlinearity by comparing the output for high versus low intensity. In the latter case, the propagation is essentially linear. If the spatial frequency of the modulation lies inside the instability window, the amplitude of the reference wave increases at the expense of the pump.

Theoretical setup. - Our theoretical model for the beam propagation incorporates the dominant dispersive and Kerr effects in a nonlinear Schrödinger equation,

$i \frac{\partial u}{\partial \zeta}=-\frac{1}{2} \nabla_{\perp}^{2} u-|u|^{2} u, \quad 0<\zeta<\tilde{l}$ (glass),

$i \frac{\partial u}{\partial \zeta}=-\frac{1}{2} \frac{n_{0}^{(1)}}{n_{0}^{(2)}} \nabla_{\perp}^{2} u-\frac{n_{2}^{(2)}}{n_{2}^{(1)}}|u|^{2} u, \quad \tilde{l}<\zeta<\tilde{L} \quad$ (air),

where space is rescaled by the wave number, $(\xi, \eta, \zeta)=$ $k^{(1)} \times(x, y, z)$, and the electric field envelope is rescaled using $u=\left(n_{2}^{(1)} / n_{0}^{(1)}\right)^{1 / 2} E$. The superscript $(j)$ denotes the medium, with $j=1$ for glass and $j=2$ for air. The Kerr coefficients of glass and air are $n_{2}^{(1)}=3.2 \times 10^{-16} \mathrm{~cm}^{2} / \mathrm{W}$ and $n_{2}^{(2)}=3.2 \times 10^{-19} \mathrm{~cm}^{2} / \mathrm{W}$, respectively. Additionally, $n_{0}^{(1)}=1.5$ and $n_{0}^{(2)}=1$. The above setting (incorporating the transmission losses at each slide) can be written compactly as

$$
i \frac{\partial u}{\partial \zeta}=-\frac{1}{2} D(\zeta) \nabla^{2} u-N(\zeta)|u|^{2} u-i \gamma(\zeta) u,
$$

where $D(\zeta)$ and $N(\zeta)$ are piecewise-constant functions in consonance with Eq. (1) and $\gamma(\zeta)$ is the loss rate. Equation (2) possesses plane wave solutions of the form

$$
u_{0}=A_{0} e^{-\int^{\zeta} \gamma\left(\zeta^{\prime}\right) d \zeta^{\prime}} e^{i A_{0}^{2} \int^{\zeta} N\left(\zeta^{\prime}\right)\left(e^{-2} \int^{\zeta^{\prime}} \gamma(\tilde{\xi}) d \tilde{\zeta}\right)} d \zeta^{\prime},
$$

where $A_{0}$ is the initial amplitude. We perform a stability analysis by inserting a Fourier-mode decomposition, $u=$ $u_{0}(\zeta)\left[1+w(\zeta) \cos \left(k_{\xi} \xi\right) \cos \left(k_{\eta} \eta\right)\right]$ (where $w=F+i B$ is a small perturbation) into Eq. (2). This yields

$$
\frac{d^{2} F}{d \zeta^{2}}=\frac{1}{D} \frac{d D}{d \zeta} \frac{d F}{d \zeta}+\left[-\frac{1}{4} \bar{k}^{4} D^{2}+N \bar{k}^{2} D\left|u_{0}\right|^{2}\right] F,
$$

where $\bar{k}^{2}=k_{\xi}^{2}+k_{\eta}^{2}$. While one can analyze Eq. (4) directly, the weak variation of $D(\zeta)$ can be exploited by substituting $D(\zeta)$ with its average and the losses at the interfaces can be ignored. [We have checked that this has little effect on the results from Eq. (4).] Under these additional simplifications, Eq. (4) is a Hill equation which for the piecewise-constant nonlinearity coefficient is the wellknown Kronig-Penney model [18]. This can be solved analytically in both glass and air (with two integration constants for each type of region). We match the solutions at the glass-air boundaries and obtain matching conditions at $\zeta=\tilde{l}$ and $\zeta=\tilde{L}$. In so doing, we employ Bloch's theorem (and the continuity of $F$ and $\frac{d F}{d \zeta}$ ), according to which $F(\zeta)=e^{-i \omega \zeta} H(\zeta)$, where $H$ is a periodic function of period $\tilde{L}$ [18]. This yields a homogeneous $4 \times 4$ matrix equation, whose solution gives the following equation for $\omega$ :

$$
\begin{aligned}
\cos (\omega \tilde{L})= & -\frac{s_{1}^{2}+s_{2}^{2}}{2 s_{1} s_{2}} \sin \left(s_{1} \tilde{l}\right) \sin \left[s_{2}(\tilde{L}-\tilde{l})\right] \\
& +\cos \left(s_{1} \tilde{l}\right) \cos \left[s_{2}(\tilde{L}-\tilde{l})\right] \\
\equiv & G(\bar{k})
\end{aligned}
$$

where $s_{1}^{2}=\bar{k}^{2} D^{(1)}\left(\bar{k}^{2} D^{(1)} / 4-N^{(1)}\left|u_{0}\right|^{2}\right) \quad$ and $\quad s_{2}^{2}=$ $\bar{k}^{2} D^{(2)}\left(\bar{k}^{2} D^{(2)} / 4-N^{(2)}\left|u_{0}\right|^{2}\right)$. Therefore, $|G(\bar{k})| \leq 1 \mathrm{im}-$ plies stability and $|G(\bar{k})|>1$ leads to MI.

Results. - Before discussing our results, it is necessary to point out two additional assumptions. First, we assume in our numerical simulations that the dynamics is effectively one dimensional (1D) along the direction of the modulation (i.e., we use $k_{\eta}=0$ and vary $k_{\xi}$ ). Accordingly, we convert the $2 \mathrm{D}$ interference patterns recorded on the CCD to $1 \mathrm{D}$ ones by integrating along the direction orthogonal to the modulation. Second, we assume that the 
modulational dynamics of the (weakly decaying) central part of the Gaussian beam of the experiment is similar to that of a plane wave with the same intensity. We tested both assumptions and confirmed them a priori through our experimental and numerical results and a posteriori through their quantitative comparison.

The input field is $u=A_{0}+\epsilon_{0} \exp \left(i k_{\xi} \xi\right)$, where $A_{0}$ and $\epsilon_{0}$ are the amplitudes of the pump and reference beams, respectively, and $\left|\epsilon_{0}\right|^{2} \ll\left|A_{0}\right|^{2}$. For linear propagation (low intensity, $I_{\mathrm{P} 2}$ ), the intensity pattern at the output of the NLM is approximately the same as that at the input; it is about $\left|A_{0}\right|^{2}+\left|\epsilon_{0}\right|^{2}+2 A_{0} \epsilon_{0} \cos \left(k_{\xi} \xi\right)$. For the nonlinear case (high intensity, $I_{\mathrm{P} 1}$ ), higher harmonics are generated and the intensity is about $\left|A_{1}\right|^{2}+2 A_{1} \epsilon_{1} \cos \left(k_{\xi} \xi\right)+$ $2 A_{1} \epsilon_{2} \cos \left(2 k_{\xi} \xi\right)+\cdots$, where $A_{1}$ and $\epsilon_{n}(n=1,2, \ldots)$ are, respectively, the amplitudes of the pump beam and the $n$th harmonic at the output of the NLM. The Fourier transform (FT) of the latter intensity is $\left|A_{1}\right|^{2} \delta\left(f_{\xi}\right)+$ $A_{1} \epsilon_{1} \delta\left(f_{\xi}-k_{\xi} / 2 \pi\right)+A_{1} \epsilon_{1} \delta\left(f_{\xi}+k_{\xi} / 2 \pi\right)+A_{1} \epsilon_{2} \delta\left(f_{\xi}-\right.$ $\left.k_{\xi} / \pi\right)+A_{1} \epsilon_{2} \delta\left(f_{\xi}+k_{\xi} / \pi\right)+\cdots$. The FT peak height ratios (first order:zeroth order), $r_{0}=\epsilon_{0} / A_{0}$ and $r_{1}=$ $\epsilon_{1} / A_{1}$, are approximately equal to the amplitude ratios of the reference and pump waves. (For the experimental value of $\epsilon_{0}=A_{0} / 10$, the error introduced by this approximation is roughly $1 \%$.) The value of $r_{1}$ increases with propagation distance as the amplitude of the reference increases. In the linear case, $r_{0}$ is constant. We use the ratio $R=r_{1} / r_{0}$ as a diagnostic measure for both our experimental and numerical results (so that $R>1$ indicates growth of the perturbation). This measurement is equivalent to the ratio $r_{1}(\zeta=\bar{\zeta}) / r_{1}(\zeta=0)$ (where $\bar{\zeta}$ is the scaled NLM length) but is more robust experimentally. In the numerical simulations, the peaks in the FT are sharp, whereas they are broader in the experiments. Thus, when computing $R$ from the experiments, we used the area under the peaks instead of the peak value.

Figure 2 shows the ratio $R(k)$ (where $k=k_{\xi} \eta_{0}^{(1)}$ is the sine of the angle between the pump and reference beams) for the structure with $2.1 \mathrm{~mm}$ air spacings. There are two instability bands (quantified by $R>1$ ) within the measurement range. The appearance of the second band is a unique feature of the layered medium that originates from the periodicity of the structure in the evolution variable. The maximum growth of the perturbation in the first and second bands appear at $k=6.0 \times 10^{-3}$ and $k=1.70 \times 10^{-2}$, with values of $R=2.05$ and $R=1.19$, respectively. The increase in the modulation is clearly visible in the $1 \mathrm{D}$ intensity patterns (see Fig. 3). The position of the instability bands is in very good agreement with both numerical and theoretical $(|G|>1)$ predictions. However, the simulation typically shows a stronger instability than the experiment. This results from the latter's 3D nature, which is not captured in the simulation. In the experiment, the spatial and temporal overlap of the two beams decreases with increasing $k$, resulting in weakening of the higher-
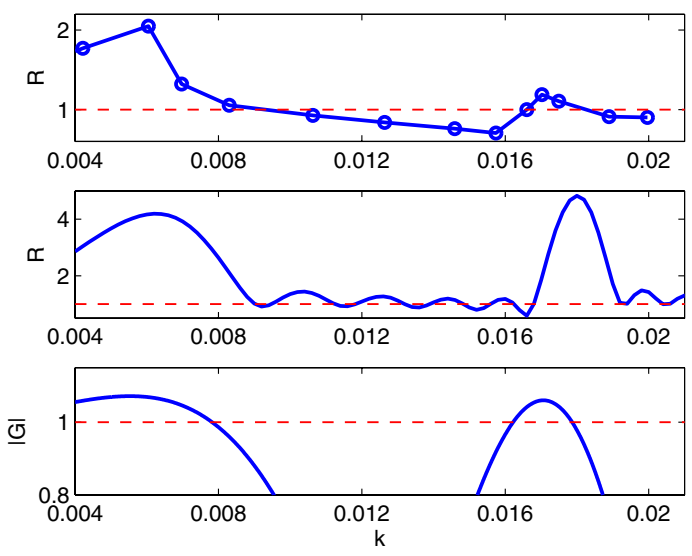

FIG. 2 (color online). Comparison of experimental (top), numerical (middle), and analytical (bottom) results for the $1 \mathrm{~mm}$ glass - $2.1 \mathrm{~mm}$ air configuration as a function of the dimensionless wave number $k$. For the diagnostics $R$ and $|G|$ (defined in the text), values larger than 1 correspond to $\mathrm{MI}$.

order peaks. The nonlinearity also has a lower aggregate strength in the experiment because of temporal dispersion. Nevertheless, the simulations successfully achieve our primary goal of quantitatively capturing the locations of the instability windows.

The top panels of Fig. 3 show the experimental normalized 1D intensity patterns at the output of the NLM for $I_{\mathrm{P} 1}$ (dashed curve) and $I_{\mathrm{P} 2}$ (solid curve). The left panels are for $k=4.2 \times 10^{-3}$ (in the first instability band), and the right ones are for $k=1.70 \times 10^{-2}$ (in the second band). The
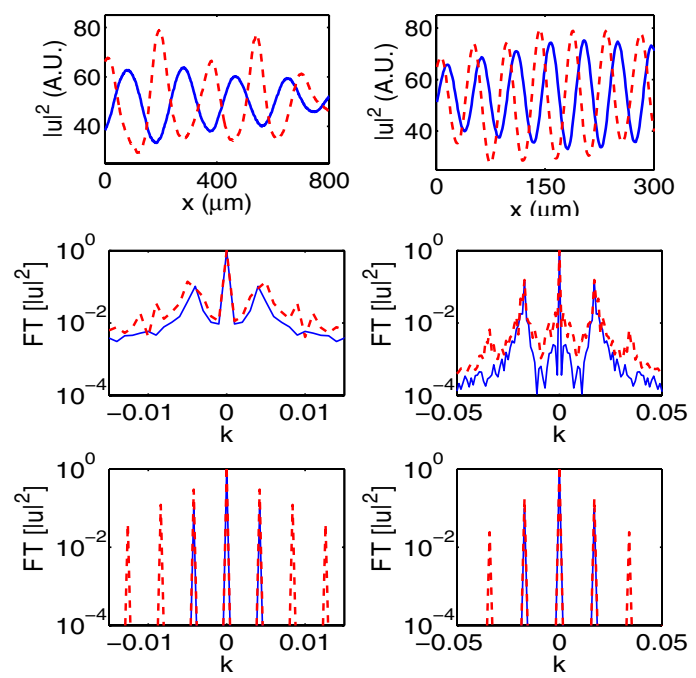

FIG. 3 (color online). Experimental normalized 1D intensity patterns (top) and Fourier spectra (middle) and numerical Fourier spectra (bottom) at the end of propagation $(z=$ $16.5 \mathrm{~mm}$ ) of the layered structure with six $1 \mathrm{~mm}$ glass slides, each pair of which sandwiches $2.1 \mathrm{~mm}$ of air. We depict the wave numbers $k=0.0042$ (first instability band; left panels) and $k=$ 0.017 (second band; right panels). The dashed (solid) curves are for high (low) intensity $I_{\mathrm{P} 1}\left(I_{\mathrm{P} 2}\right)$. 

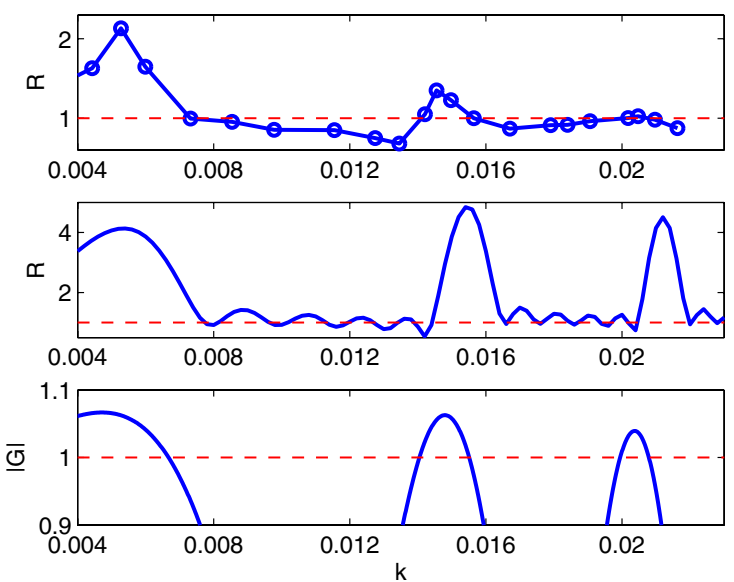

FIG. 4 (color online). Same as Fig. 2 but for the $1 \mathrm{~mm}$ glass$3.1 \mathrm{~mm}$ air configuration. Here there is a third MI band.

amplitude of the modulation increases for high intensity cases due to MI. We have also observed in the FT of the experimental intensity patterns (middle panels of Fig. 3) the appearance of higher spatial harmonics of the initial modulation in the instability regions. The first-order peaks (the ones closer to $k=0$ ) correspond to the modulation of the input beam and are present for both low and high intensity. The harmonics correspond to the narrowing of the peaks in the spatial interference pattern and appear only for high intensity. Strong harmonics are expected only within the instability regions. We also observed this harmonic generation in numerical simulations (bottom panels of Fig. 3), in good agreement with the experiments. In contrast, we did not observe such harmonics in the experiments for $k$ values in the stable regions $(R<1$ in Fig. 2), again in agreement with theory.

Figure 4 shows the instability windows for a structure with a different periodicity (with $3.1 \mathrm{~mm}$ air between each pair of $1 \mathrm{~mm}$ glass slides). The instability bands shift towards lower $k$ and the longer spatial period in the structure results in a smaller spacing between the instability bands in Fourier space. The peaks of the first two bands are at $k=5.3 \times 10^{-3}$ and $k=1.46 \times 10^{-2}$, respectively, and a third band appears around $k=2.05 \times 10^{-2}$. Once again, we obtain good agreement between experiment, numerics, and theory. It is not straightforward to give an intuitive explanation of the precise locations and widths of the stability bands or instability zones [beyond employing the simple transcendental expression of Eq. (5)]. However, by examining simplified cases (e.g., very narrow air gaps between wide glass slides or vice versa) [18], one can extract useful information, such as the decreasing width of the MI zones for increasing zone index (which can be seen, e.g., in Figs. 2 and 4).

Conclusions. - We provided the first experimental realization of MI in a medium periodic in the evolution variable. The linear stability analysis of the pertinent plane waves led to an effective Kronig-Penney model, which was used to describe the instability bands quantitatively, providing a direct association of the MI bands with the latter's forbidden energy zones. One of the hallmarks of the periodic medium is the presence of additional MI bands as opposed to the single band that occurs in uniform media. We found very good agreement between our theoretical predictions for the modulationally unstable bands and those obtained experimentally and numerically. We also observed higher spatial harmonics for modulationally unstable beams (another characteristic trait of MI). Many interesting extensions are possible, including the study of solitary waves that result from MI in layered Kerr media.

We acknowledge support from the DARPA Center for Optofluidic Integration (D.P.), the Caltech Information Science and Technology initiative (M.C., M.A.P.), and NSF-DMS and CAREER (P. G. K.).

[1] T. B. Benjamin and J.E. Feir, J. Fluid Mech. 27, 417 (1967).

[2] L. A. Ostrovskii, Sov. Phys. JETP 24, 797 (1967).

[3] T. Taniuti and H. Washimi, Phys. Rev. Lett. 21, 209 (1968); A. Hasegawa, Phys. Rev. Lett. 24, 1165 (1970).

[4] P. G. Kevrekidis and D. J. Frantzeskakis, Mod. Phys. Lett. B 18, 173 (2004).

[5] K. E. Strecker et al., Nature (London) 417, 150 (2002).

[6] L. Salasnich et al., Phys. Rev. Lett. 91, 080405 (2003); L. D. Carr and J. Brand, Phys. Rev. A 70, 033607 (2004).

[7] S. Wabnitz, Phys. Rev. A 38, 2018 (1988).

[8] M. Soljačić and J.D. Joannopoulos, Nat. Mater. 3, 211 (2004).

[9] J. Meier et al., Phys. Rev. Lett. 92, 163902 (2004).

[10] A. Smerzi et al., Phys. Rev. Lett. 89, 170402 (2002); F. S. Cataliotti et al., New J. Phys. 5, 71 (2003); B. Wu and Q. Niu, Phys. Rev. A 64, 061603 (2001); V. V. Konotop and M. Salerno, Phys. Rev. A 65, 021602 (2002).

[11] B. A. Malomed, Prog. Opt. 43, 71 (2002); S. K. Turitsyn et al., C.R. Physique 4, 145 (2003).

[12] I. Towers and B. A. Malomed, J. Opt. Soc. Am. B 19, 537 (2002).

[13] M. Centurion et al., Phys. Rev. Lett. 97, 033903 (2006).

[14] P. G. Kevrekidis et al., Phys. Rev. Lett. 90, 230401 (2003); see also the review B. A. Malomed, Soliton Management in Periodic Systems (Springer, Berlin, 2006).

[15] N. J. Smith and N. J. Doran, Opt. Lett. 21, 570 (1996); F. Kh. Abdullaev et al., Phys. Lett. A 220, 213 (1996).

[16] Z. Rapti et al., Phys. Scr. T107, 27 (2004).

[17] W. Magnus and S. Winkler, Hill's Equation (Wiley, New York, 1966).

[18] C. Kittel, Introduction to Solid State Physics (John Wiley and Sons, New York, 1986); see also I. I. Gol'dman and V.D. Krivchenkov, Problems in Quantum Mechanics (Dover, New York, 1993), pp. 59-61.

[19] D. Hennig et al., Appl. Phys. Lett. 64, 2934 (1994); Yu. Gaididei et al., Phys. Rev. B 55, R13365 (1997). 